\title{
Maeve McCusker, Patrick Chamoiseau. Recovering \\ Memory
}

\section{Luigia Pattano}

\section{Q OpenEdition \\ 1 Journals}

\section{Edizione digitale}

URL: http://journals.openedition.org/studifrancesi/8112

DOI: $10.4000 /$ studifrancesi.8112

ISSN: 2421-5856

\section{Editore}

Rosenberg \& Sellier

\section{Edizione cartacea}

Data di pubblicazione: 1 juillet 2009

Paginazione: 453-454

ISSN: 0039-2944

\section{Notizia bibliografica digitale}

Luigia Pattano, «Maeve McCusker, Patrick Chamoiseau. Recovering Memory», Studi Francesi [Online], 158 (LIII | II) | 2009, online dal 30 novembre 2015, consultato il 13 janvier 2021. URL: http://

journals.openedition.org/studifrancesi/8112 ; DOI: https://doi.org/10.4000/studifrancesi.8112

Questo documento è stato generato automaticamente il 13 janvier 2021.

\section{(c) (i) $\odot$}

Studi Francesi è distribuita con Licenza Creative Commons Attribuzione - Non commerciale - Non opere derivate 4.0 Internazionale. 


\title{
Maeve McCusker, Patrick Chamoiseau. Recovering Memory
}

\author{
Luigia Pattano
}

\section{NOTIZIA}

MAEVE MCCUSKER, Patrick Chamoiseau. Recovering Memory, Liverpool, Liverpool University Press, 2007, pp. 184.

1 Al centro del pensiero e delle manifestazioni culturali del Novecento europeo a partire dal secondo dopoguerra, la memoria assume una rilevanza e un peso inediti nel contesto caraibico contemporaneo, caratterizzato da "a paradox of reluctant amnesia» nei confronti della drammatica storia coloniale e schiavista, che fa sì che «any attempt to recover collective memory will also, by definition, be an attempt to recover from that very memory» (p. 9). A differenza dei traumi collettivi provocati, per esempio, dall'Olocausto e dalla guerra in Vietnam, donde è scaturita una vasta letteratura (e, in un secondo tempo, anche una critica) della memoria basata sulle testimonianze dei sopravvissuti (living memory), le ombre del passato caraibico hanno prodotto, a partire dagli anni Ottanta del Novecento, una letteratura ossessionata dalla necessità (e insieme dalla consapevolezza dell'impossibilità) di creare - attraverso la fantasia - le condizioni per la formazione di una memoria collettiva. Nell'ambito della produzione letteraria antillese di lingua francese, l'opera di Patrick Chamoiseau, cui questo tema è particolarmente caro, è letta da Maeve McCusker come tentativo fondamentalmente politico di esplorare «the compelling momentum of modernization in the Caribbean, while simultaneously recreating the culture of the past» (p. 18). Obiettivo di questo saggio monografico è dunque quello di analizzare l'interazione tra linguaggio e politica, tra etica ed estetica, in un'opera di indubbio valore quale è quella di Chamoiseau che, unendo ambizione letteraria e attività di resistenza, partecipa in maniera manifesta al progetto di elaborazione di una «highly distinctive memorial literature» (p.18). Felice risulta la scelta del critico di analizzare i testi letterari in esame (tutta la produzione narrativa di Chamoiseau da Chronique des sept misères a Biblique des derniers gestes, 
compreso Écrire en pays dominé) alla luce degli studi postcoloniali e delle teorie sulla memoria e sul trauma elaborate a proposito dell'Olocausto. Quest'ottica pare legittimata dalle consonanze considerevoli individuate da McCusker tra la politica identitaria creolista, fortemente circoscritta, e i dibattiti mondiali sulla memoria. L'analisi puntuale e cronologica dei testi porta il critico ad avanzare una proposta di lettura dell'opera dell'autore martinicano come transizione da un'estetica del ricordare e del trascrivere a una poetica della materialità, evidente in Biblique des derniers gestes, dove il corpo emerge come archivio e testimone attivo della memoria. 